\title{
Mandenkan
}

MANDENIKAN Bulletin semestriel d'études linguistiques mandé

49 | 2013

Le maninka du Niokolo

\section{Particules adnominales}

Section 15

Denis Creissels

\section{(2) OpenEdition}

Journals

Édition électronique

URL : https://journals.openedition.org/mandenkan/611

DOI : 10.4000/mandenkan. 611

ISSN : 2104-371X

Éditeur

Llacan UMR 8135 CNRS/Inalco

Édition imprimée

Date de publication : 1 juin 2013

Pagination : 98-99

ISSN : 0752-5443

Référence électronique

Denis Creissels, «Particules adnominales », Mandenkan [En ligne], 49 | 2013, mis en ligne le 25 avril

2014, consulté le 16 janvier 2023. URL : http://journals.openedition.org/mandenkan/611 ; DOI :

https://doi.org/10.4000/mandenkan.611

Ce document a été généré automatiquement le 16 janvier 2023.

\section{(c) $)(1)(2)$}

Creative Commons - Attribution - Pas d'Utilisation Commerciale - Partage dans les Mêmes Conditions 4.0 International - CC BY-NC-SA 4.0

https://creativecommons.org/licenses/by-nc-sa/4.0/ 


\section{Particules adnominales}

\section{Section 15}

\section{Denis Creissels}

1 Les particules adnominales suivantes ont été relevées :

• dámmáa 'à part', ‘quant à eux', ‘entre eux', (postposé au constituant nominal)

\begin{tabular}{|l|l|l|l|l|l|l|l|}
\hline (143) & a. & Músu & mee & náa & $a$ & ñáyǎa & to, \\
\hline & & femme & POTN & venir & 35 S & fête.D & Loc \\
\hline & & 'Les femmes n'assistent pas à la cérémonie en question, \\
\hline & b. & kẽe & dámmáa & doroy & née & se & náa. \\
\hline & & homme & à_part & seulement & FOc & POTP & venir \\
\hline & \multicolumn{6}{|l}{} \\
\hline
\end{tabular}

\begin{tabular}{|l|l|l|l|l|l|l|l|l|}
\hline$(144)$ & Ń-te & lu & dámmáa & se & á & $k e$ & wóo & ñăa. \\
\hline & 1 PL-EMPH & PL & à_part & POTP & 3SG & faire & DEM & manière.D \\
\hline
\end{tabular}

- doroy 'seulement' (postposé au constituant nominal, souvent suivi de la particule de focalisation lée)

\begin{tabular}{|l|l|l|l|l|l|l|}
\hline$(145)$ & Kẽe & & lu & dorop & née & náa-ta. \\
\hline & homme.D & PL & seulement & FOC & venir-ACPP & \\
\hline
\end{tabular}


'Seuls les hommes sont venus.'

- dúg particule contrastive (postposé au constituant nominal)

\begin{tabular}{|l|l|l|l|l|l|l|l|}
\hline (146) & I-te & dún, & $i$ & be & mún & née & kee-kán? \\
\hline & 2SG-EMPH & CONTR & 2SG & COPLOC & quoi & FOC & faire-PROG \\
\hline & 'Et toi, qu'est-ce que tu es en train de faire?' \\
\hline
\end{tabular}

- $f^{2} a$ (o) particule intensive (postposé au constituant nominal), dont la combinaison avec les pronoms personnels a déjà été évoquée en 11.3.

\begin{tabular}{|l|l|l|l|l|l|l|l|}
\hline$(147)$ & $\eta$ & baabá & fắno & lée & ye & á & fo. \\
\hline & $1 S \mathrm{SG}$ & papa & INT & FOC & ACPP & 3SG & dire \\
\hline \multicolumn{7}{|c|}{ 'C'est mon père lui-même qui a dit ça.' } \\
\hline
\end{tabular}

- fanay 'aussi', 'à son tour' (postposé au constituant nominal)

\begin{tabular}{|l|l|l|l|l|l|l|l|}
\hline (148) & a. & I-té & fanay & bé & taya-la & lée & baa? \\
\hline & & 2SG-EMPH & aussi & COPLOC & partir-INF & FOC & Q \\
\hline & & \multicolumn{6}{|c|}{ 'Est-ce que tu vas partir toi aussi ?' } \\
\hline
\end{tabular}

\begin{tabular}{|l|l|l|l|l|l|l|l|l|l|}
\hline & b. & Mus-óo & lu & fanay & sé & naam-óo & lu & $k e$ & lée. \\
\hline & & femme-D & PL & aussi & POTP & coutume-D & PL & faire & FOC \\
\hline & \multicolumn{6}{|c|}{ 'Les femmes aussi font leurs cérémonies coutumières.' } \\
\hline
\end{tabular}

\begin{tabular}{|l|l|l|l|l|l|l|l|l|l|}
\hline & c. & Gúlutaaláa & lu & fanay & se & sólimáa & lu & táa \\
\hline & & gouloutala.D & PL & aussi & POTP & solima.D & PL & prendre \\
\hline & & Les gouloutalas à leur tour prennent les solimas \\
\hline & ká & a & kárafá & kamarín-o & lu & la. & \\
\hline & & INF & $2 S G$ & confier & kamaring-D & PL & OBL & \\
\hline & pour les confier aux kamarings. \\
\hline
\end{tabular}

• háani, háari 'même' (antéposé au constituant nominal) 


\begin{tabular}{|l|l|l|l|l|l|l|l|l|}
\hline (149) & a. & A & te & máninka-káy-o & móyi-la & háari & dondí & na. \\
\hline & & 3 SG & COPN & maninka-langue-D & entendre-INF & même & un_peu & OBL \\
\hline & \multicolumn{6}{|c|}{ 'Il ne comprend pas le maninka, même un peu.' } \\
\hline
\end{tabular}

\begin{tabular}{|l|l|l|l|l|l|l|}
\hline & b. & Háari & dindìy-o & ye & ñì & loy. \\
\hline & & même & enfant-D & ACPP & DEM & savoir \\
\hline & & \multicolumn{3}{|l}{} \\
\hline
\end{tabular}

• kéy 'vraiment', ‘en tout cas' (postposé au constituant nominal)

\begin{tabular}{|l|l|l|l|l|l|}
\hline (150) & Wóo & kén & diyáa-ta & $y$ & yẹ. \\
\hline & DEM & en_tout_cas & plaire-ACPP & 1 SG & BEN \\
\hline & 'Ça en tout cas ça me plaît.' \\
\hline
\end{tabular}

2 Kéy apparait notamment dans l'expression niy wóo kéy téy (prononcée nǒokéntéy) 'sinon' (litt. 'si vraiment ce n'est pas cela')

- lée particule de focalisation (se postpose au constituant nominal sans entraîner aucun déplacement du constituant concerné)

\begin{tabular}{|l|l|l|l|l|l|l|}
\hline$(151)$ & a. & Dindin-o & lée & náa-ta & ñins-óo & ti. \\
\hline & & enfant-D & FOC & venir-ACPP & vache-D & ОВL \\
\hline & & \multicolumn{4}{|l}{} \\
\hline
\end{tabular}

\begin{tabular}{|l|l|l|l|l|l|l|}
\hline & b. & Dindin-o & náa-ta & ñins-óo & lée & ti. \\
\hline & & enfant-D & venir-AcPP & vache-D & FOC & ОВL \\
\hline & & \multicolumn{3}{|l}{} \\
\hline
\end{tabular}

3 Sur lée, cf. aussi 21.1. 


\section{AUTEUR}

DENIS CREISSELS

Université de Lyon

Denis.Creissels@univ-lyon2.fr 